\title{
Histologic Characterization of Human Scarred Vocal Folds
}

\section{$\operatorname{AUTHOR}(S)$ :}

Hirano, Shigeru; Minamiguchi, Sachiko; Yamashita, Masaru; Ohno, Tsunehisa; Kanemaru, Shin-ichi; Kitamura, Morimasa

\section{CITATION:}

Hirano, Shigeru ...[et al]. Histologic Characterization of Human Scarred Vocal Folds. Journal of Voice 2009, 23(4): 399-407

ISSUE DATE:

2009-07

URL:

http://hdl.handle.net/2433/84841

\section{RIGHT:}

c 2009 The Voice Foundation.; この論文は出版社版でありません。引用 の際には出版社版をご確認ご利用ください。; This is not the published version. Please cite only the published version. 


\title{
Histologic Characterization of Human Scarred Vocal Folds
}

(Abbreviation: Human scarred vocal folds)

\author{
Shigeru Hirano, MD., PhD. \\ Sachiko Minamiguchi, MD., PhD.* \\ Masaru Yamashita, MD., PhD.** \\ Tsunehisa Ohno, MD. PhD.*** \\ Shin-ichi Kanemaru, MD., PhD. \\ Morimasa Kitamura, MD.****
}

Department of Otolaryngology-Head and Neck Surgery, Kyoto University Graduate School of Medicine, Kyoto, Japan

*Laboratory of Anatomic and Clinical Pathology, Kyoto Medical Center, Kyoto, Japan

** Division of Otolaryngology Head and Neck Surgery, University of WisconsinMadison

***Department of Otolaryngology Head and Neck Surgery, Vanderbilt School of Medicine

****Department of Otolaryngology, Kyoto Medical Center, Kyoto, Japan

\section{This paper was presented at Annual Symposium of The Voice Foundation in} Philadelphia in 2007.

\section{Correspondence to:}

Shigeru Hirano, MD, Department of Otolaryngology-Head \& Neck Surgery, Kyoto University Graduate School of Medicine, Sakyo-ku, Kyoto 606-8507, JAPAN Tel: +81-75-751-3346, Fax:+81-75-751-7225, E-mail: hirano@ent.kuhp.kyoto-u.ac.jp 


\section{ABSTRACT}

Objectives: Vocal fold scarring remains a significant problem. Although several animal models have been developed to improve our understanding of the histopathology, the histologic features of scarred human vocal folds have rarely been reported. The present case studies aimed to define the histologic changes of scarred human vocal folds caused by cordectomy or cordotomy. Methods: Ten patients with the scarred vocal folds were involved in this study. Nine patients with early glottic cancer underwent endoscopic cordectomy, and one patient underwent superficial cordotomy for idiopathic scar. The post-cordectomy or cordotomy scar was biopsied or resected 3 to 13 months after the original procedure. After confirming absence of any tumor in cancer patients, the remaining specimens were used in the present study. Histologic examination investigated deposition of extracellular matrix (ECM) including collagen, elastin, hyaluronic acid (HA), fibronectin, and decorin in the lamina propria of the scarred vocal folds.

Results: There was a wide range variation in the deposition of ECM in scarred vocal folds. Excessive and disorganized collagen deposition was observed in most cases that had undergone deep resection of the lamina propria, whereas deposition of collagen was mild and well organized after superficial resection. Decorin was retained in all cases after superficial cordectomy or cordotomy, but varied after deep resection. Deposition of elastin, HA, and fibronectin varied regardless of depth of injury.

Conclusion: Histology of scarred vocal folds may vary with degree of injury and individual healing mechanism.

Key words: vocal fold scarring, human, histology, collagen, hyaluronic acid, decorin 


\section{INTRODUCTION}

Vocal fold scarring occurs after repeated phonotrauma or inflammation, and it causes a marked decrease in vibration of the vocal fold mucosa ${ }^{1,2}$, resulting in a hoarse, harsh, and weak voice. Repeated phonotrauma often occurs in professional vocalists, such as singers and actors, and after the vocal fold has become scarred, the career of such professionals is threatened. Vocal fold scarring also affects patients who received endoscopic laser surgery for early glottic cancer. Recent advances in laser surgery techniques have improved the local control of early glottic cancers, but postoperative scarring is inevitable. Severity of scarring may depend on extent of tissue resection. Vocal fold scarring is characterized by histologic changes leading to an increase of mucosal stiffness and reduction of traveling waves during phonation. To date, the optimum treatment has not been established, and the management of scarred vocal folds remains a challenge.

It is important to understand the histologic changes underlying scarring in order to develop any effective methods for restoring tissue architecture to a normal or near-normal state. Several animal studies have reported important findings, including dense or disorganized collagen deposits, decrease or total loss of elastin, increase of fibronectin, and decrease of decorin and fibromodulin $^{3-12}$. Variance in results is probably due to differences in animal used, time after injury, and type of injury. Wound healing may differ among animal species, tissues, and injury mechanism. In particular, primary histologic architecture of the vocal fold varies among species. Unlike other mammals, human vocal folds have a well-defined three-layer structure in the lamina propria consisting of superficial, intermediate, and deep layers ${ }^{13}$. The superficial layer contains various proteoglycans and glycosaminoglycans, while the intermediate layer chiefly consists of elastin and the deep layer is composed mainly of collagen. The intermediate and deep layers form the vocal ligament, a structure not found in other mammals. Therefore, the histologic changes of scarred human vocal folds may be different from those seen in other animals, and thus 
human studies are necessary. Because it is difficult to develop a human model of vocal fold scarring, however, rare reports on the histology of scarred human vocal folds have been published.

At Kyoto University and Kyoto medical center (Japan), we perform endoscopic laser surgery for early glottic cancer and carry out planned or eventual endoscopic laryngeal revision after the initial procedure in cases with close margin or suspicion of recurrence. During the second procedure, we biopsy scarred vocal folds to detect residual tumor. In the present study, we examined histologic changes of scarred human vocal folds using biopsy tissues obtained at endoscopic revision surgery for glottic cancer. An additional case with idiopathic scarred vocal folds was studied. 


\section{MATERIALS AND METHODS}

\section{Patients and Endoscopic Procedures}

Ten patients enrolled in this study. Nine cases (all male, 54-76 years old) underwent endoscopic laser surgery for T1 glottic squamous cell carcinoma at Kyoto University Hospital or Kyoto Medical Center. Informed consent was obtained from each patient after full explanation of this study, which had been approved by the institutional review board. The method of cordectomy was classified according to the system of the Working Committee of the European Laryngological Society, ${ }^{14}$ in which type I is subepithelial cordectomy, type II is subligamental cordectomy, type III is transmuscular cordectomy, type IV is total cordectomy, and type V is extended cordectomy. As indicated in Table I, we performed type I cordectomy for four patients, type II for four patients, and type III for one patient. In all cases, the unilateral vocal fold was treated with a potassium titanyl phosphate (KTP) laser at an output of 5 to $8 \mathrm{~W}$ under direct laryngoscopy. Case 4 underwent postoperative radiotherapy following type I cordectomy.

Revision endoscopic surgery was performed 3-13 months postoperatively for all nine patients due to close margin or possible recurrence. At each time point, the primary laser wound had healed and had been covered by regenerated epithelium. Under direct laryngoscopy, the vocal fold was biopsied at the scarred site. Biopsied tissues included the epithelium and almost the whole layer of the lamina propria. After confirming that there were no tumor cells by routine histologic examination, the remnant tissue from each specimen was subjected to the present study.

One additional patient (Case 10) with scarred vocal folds after cordotomy was also examined. The original procedure was done at another hospital for idiopathic scarred vocal fold, probably due to involvement of rheumatoid arthritis. She was referred to us because her postcordotomy voice had not improved. 


\section{Histologic Examination}

The tissues were fixed in 10\% formaldehyde and embedded in paraffin to investigate the distribution of collagen, elastin, hyaluronic acid (HA), fibronectin, and decorin. Eight micron thick sections were prepared for histologic examination. Elastica-van-Gieson staining was used to identify collagen and elastin, and Alcian blue staining was used for HA after digestion with hyaluronidase. This technique was developed by Gray et al. ${ }^{15}$ to correctly identify HA in the vocal fold lamina propria. To perform hyaluronidase digestion, $50 \mathrm{mg}$ of bovine testicular hyaluronidase (Sigma, St Louis, MO) was diluted in $100 \mathrm{~mL}$ of phosphate-buffered saline (PBS) and sections were incubated in the PBS-hyaluronidase mixture for 1 hour at $37^{\circ} \mathrm{C}$. Then the sections were stained with Alcian blue ( $\mathrm{pH}$ 2.5). HA was detected by comparing sections with and without digestion by hyaluronidase.

Immunohistochemistry was used to detect fibronectin and decorin. Monoclonal antibodies targeting fibronectin and decorin were obtained from Takara Bio Inc. (Japan), and R\&D Systems Inc. (Minneapolis, MN), respectively. Slides were treated with 3\% hydrogen peroxide and then with protein blocking reagent (DAKO, Carpinteria, CA), followed by incubation with antibody, at the concentration of $25 \mu \mathrm{g} / \mathrm{mL}$ for both of fibronectin and decorin,

for 30 minutes at room temperature. The peroxidase-antiperoxidase technique with diaminobenzidine as the substrate was employed to assess the expression of each molecule (positive regions were stained brown). Human skin tissue was used as the positive control.

Each slide was judged by 2 examiners (1 laryngologist (S.H.) and 1 pathologist (S.M.)) in a blinded fashion to detect presence and distribution of each ECM component. Since no normal control was obtained from the same individuals, the deposition of collagen was subjectively assessed by "absent", "mild", or "excessive". Deposition of elastin was assessed by "present, organized", "present, disorganized", or "absent". Depositions of HA, fibronectin, and decorin were described by "present" or "absent". For inter- and intra-rater reliability of 
measurements, all slides were re-measured. Correspondence rates were examined

between initial and repeated measures, revealing good correspondence rates of more than $95 \%$. 


\section{RESULTS}

Type I cordectomy scar

Figure 1 shows the histology of Case 1, with type I post-cordectomy scar. Collagen was deposited in a well-organized fashion throughout the lamina propria. The density of collagen was thinner in the superficial portion than in the deep portion. Elastin was well organized in the deeper portion. These findings suggest that layer structure was maintained to some degree in terms of order of distribution of fibrous proteins. However, HA was absent in the lamina propria, although fibronectin and decorin were present. It was impossible for us to determine whether or not molecules were increased or decreased in density within scar tissue because we did not have normal controls for comparison purposes. Figure 2 represents another case (Case 2) of type I post-cordectomy scar. Collagen deposition was minimal in the superficial portion of the lamina propria. HA was present throughout the lamina propria. Fibronectin was not observed, but decorin was present.

Type I cordectomy + radiotherapy

Case 4 underwent type I cordectomy followed by radiotherapy. Histology seven months after radiotherapy showed excessive collagen deposition in the superficial portion of the lamina propria (Fig. 3). Collagen was disorganized, forming thick bundles. Elastin, HA, and fibronectin were absent, but staining for decorin was slightly positive.

\section{Type II cordectomy scar}

Figure 4 shows the histology of Case 8, with type II cordectomy. Excessive disorganized collagen deposition was found throughout the lamina propria. There was no staining for elastin, HA, fibronectin, or decorin.

Type III cordectomy scar 
The histology of type III post-cordectomy scar (Case 9) showed severely disorganized collagen deposition in thick bundles throughout the lamina propria (Fig. 5). Elastin, HA, fibronectin, and decorin were barely observed.

Reumatoid arthritis (RA) + post-superficial cordotomy scar

Case 10 had idiopathic scar, possibly due to involvement of rheumatoid arthritis into the vocal fold. She had undergone superficial cordotomy by a prior surgeon to free the scar tissue. After postoperative voice had not improved three months after cordotomy, she was referred to us. She was nearly aphonic, and there was no mucosal vibration on stroboscopic examination. We performed dissection of scar by elevating a microflap and resecting as much scar tissue beneath the epithelium as possible. Figure 6 shows the histology of the scar tissue, with excessive disorganized collagen deposition forming thick bundles accompanied by loss of elastin and HA. Fibronectin and decorin were present.

Summary of histology (Table II)

Among patients with type I cordectomy, two of four cases presented with mild deposition of collagen in the superficial portion of the lamina propria. Deposition of elastin, HA, and fibronectin varied among individuals, while decorin was preserved in all cases. All four Type II cordectomy patients had excessive and disorganized collagen deposition, forming thick bundles, throughout the lamina propria. Elastin disappeared or was disorganized. Deposition of HA, fibronectin, and decorin varied among individuals. The patient who had undergone type III cordectomy had excessive and disorganized collagen, forming thick bundles, throughout the lamina propria. Elastin remained, but was disorganized. Other extracellular matrix molecules such as HA, fibronectin, and decorin were not detected. 
Human scarred vocal folds 10

\section{DISCUSSION}

Vocal fold scarring has several causes, including chronic inflammation due to mechanical or chemical injury and direct injury to the vocal fold mucosa such as laser cordectomy for early glottic cancers or excessive phonomicrosurgical procedures even for benign lesions. The present preliminary study reported histological features of human scarred vocal folds caused by direct injury (cordectomy/cordotomy). The results indicate a wide range of variation in histology, probably due to the degree of damage, time point after surgery when the scar was examined, and, possibly, some difference in mechanism of wound healing.

In animal studies, collagen has been reported to be increased and/or disorganized in scarred lamina propria. The present study found less collagen deposition in the superficial portion of the lamina propria in some cases following shallow cordectomy (type I), whereas disorganized collagen deposition with thick bundles was always observed throughout the lamina propria after deeper cordectomy (type II and III). Although tissue property of the vocal fold is thought to be determined by various extracellular matrix components, it is at least speculated that thick, disorganized collagen bundles may affect tissue property. Severely disorganized collagen was observed even in type I post-cordectomy scar (Case 4), the patient who had received postoperative radiotherapy. This finding may support the previously postulated notion that radiotherapy for early glottic cancer causes fibrosis in vocal fold tissue. ${ }^{16}$

Decorin was found to be preserved, more or less, in all cases with type I postcordectomy scar, while it varied after deeper cordectomy procedures. Decorin is a small chain proteoglycan, which is normally distributed in the superficial layer of the lamina propria of human vocal folds. ${ }^{17}$ Decorin acts to regulate collagen fibrils and also inhibits formation of collagen fibers $^{15}$, suggesting that decorin may reduce scar effects. A rabbit study reported a decrease of decorin in two-month scars of rabbit vocal folds. ${ }^{10}$ It is possible that the presence of decorin in human vocal fold scars after shallow cordectomy may be a positive event for vocal fold properties. 
Deposition of other ECM components such as elastin, HA, and fibronectin varied individually regardless of depth of procedural injury. Elastin is normally found in the intermediate layer of the human vocal fold ${ }^{13}$ and contributes to maintaining appropriate elasticity of vocal fold tissue. Previous animal studies have shown reduction or absence of elastin in scarred vocal folds. In the present study, elastin was preserved and organized in the only case with type I cordectomy, while it had disappeared or was disorganized in cases with more extensive cordectomy.

HA is believed to be one of the most important molecules for maintaining ideal viscoelasticity of the vocal fold mucosa. ${ }^{15}$ It is one of the glycosaminoglycans that are widely distributed in the superficial layer of the lamina propria. It has been confirmed that HA contributes to fetal scarless wound healing, ${ }^{18}$ which suggests that HA may have a potential role in treatment and prevention of scarring. ${ }^{19}$ In a rabbit model of acute vocal fold scarring, HA levels were significantly reduced up to 15 days following injury. ${ }^{9}$ On the other hand, in a chronic scarring model of the vocal fold, the density of HA in the scarred vocal folds seemed to vary among animal studies. In rabbit and canine studies, HA density showed no significant difference in scar compared with normal vocal folds at both two months and six months, ${ }^{7}$ whereas HA was found to be significantly reduced in scarred vocal folds with a three-month rat model. ${ }^{12}$ These results indicate variations of HA due to species differences and time between insult and evaluation of scar. The current human study also demonstrated individual differences in preservation of HA in scarred vocal folds regardless of cordectomy type. It was difficult to evaluate whether or not the level of the remaining HA was normal or lower or even higher than normal, because of lack of a normal control.

Fibronectin is a large glycoprotein that acts as an adhesion molecule with binding sites for several other matrix components. The role of fibronectin in wound healing is well known. ${ }^{20}$ Fibronectin contributes to adhesion, migration, and chemotaxis of fibroblasts and also to organization of the matrix. It promotes migration of fibroblasts into the wound bed, and these 
fibroblasts produce a considerable amount of fibronectin, which leads to organization of granulation tissue in cooperation with other matrix components. When granulation tissue is replaced by neo-dermis over time, fibronectin decreases and type I collagen replaces the matrix. It is also thought that fibronectin does not disappear if the granulation tissue reaction persists, as in the cases of hypertrophic scars and keloids. Our previous canine study revealed a significant increase of fibronectin in the lamina propria of scarred vocal folds at both two and six months after injury, ${ }^{8}$ which might imply that replacement of fibronectin by other matrix components such as collagen was not completed at that time point. The present human study demonstrated the presence of fibronectin in three patients regardless of type of injury, while fibronectin disappeared in other cases, showing inter-individual variations.

The etiology of scar in Case 10 is complicated. She had been diagnosed elsewhere with idiopathic scar, possibly secondary to chronic inflammation due to rheumatoid arthritis. It is well known that rheumatoid arthritis occasionally involves the cricoarytenoid joint. ${ }^{21}$ However, it has been unclear whether or not it affects the vocal fold mucosa, although recent research has reported possible involvement of the underlying autoimmune/ inflammatory process into the vocal fold mucosa, manifested as nodule or bamboo vocal fold. ${ }^{22}$ Persistent inflammation might have caused scarring in the present case. The histology after superficial cordotomy indicated deposition of thick collagen bundles with a loss of HA.

Several factors may influence the stiffness of scarred vocal fold tissue. Particularly in the skin, collagen is thought to be primarily responsible for tissue tensile strength. ${ }^{23}$ Disorganized collagen and elastin fibers have been suggested to play an important role in increasing stiffness. ${ }^{6}$ A possible role of excessive fibronectin matrix in vocal fold stiffness was also suggested by a report associating fibronectin expression with reduced mucosal wave in human vocal fold polyps or Reinke's edema. ${ }^{24}$ The present study cannot address this aspect because of lack of normal controls and because of the small number of subjects. Further study may be needed to clarify this point. 
Another problem is that the current study used scar tissues taken at different time points because the biopsy was performed depending on clinical necessity for each patient. The correlation of timing with clinical outcome after initial procedure may be the key limitation in human study at this time.

\section{CONCLUSION}

The present case studies attempted to clarify histologic alterations in human scarred vocal folds using biopsies taken from patients who had undergone cordectomy or cordotomy. The conclusion is limited because of the small number of subjects and the lack of normal controls for histologic examination. However, it is at least suggested that there is wide inter-individual variation in histology of human scarred vocal folds. It is also suggested that deeper injury to the vocal fold may cause excessive collagen deposition forming thick bundles throughout the lamina propria, as well as less deposition of decorin compared with that observed with shallow injuries. Elastin, hyaluronic acid (HA), and fibronectin showed individual variation regardless of depth of initial injury.

\section{ACKNOWLEDGEMENT}

We appreciate Tetsuya Ohgami, Satoshi Kurokawa, Haruki Kishita, and Hitoshi Saeki, cytotechnician in Kyoto Medical Center, Japan, for their cooperation in histologic stainings. 
Human scarred vocal folds 14

\section{BIBLIOGRAPHY}

1. Benninger MS, Alessi D, Archer S, et al. Vocal fold scarring: Current concepts and management. Otolaryngol Head Neck Surg 1996;115:474-482.

2. Hirano S. Current treatment of vocal fold scarring. Curr Opin Otolaryngol Head Neck Surg. 2005; 13:143-7.

3. Hirano M. Phonosurgery. Basic and clinical investigation. Otologia (Fukuoka) $1975 ; 21$ (suppl 1): 299-303.

4. Rogerson AR, Clark KF, Bandi SR, Bane B. Voice and healing after vocal fold epithelium removal by CO2 laser vs. microlaryngeal stripping. Otolaryngol Head Neck Surg $1996 ; 115: 352-359$.

5. Blakeslee DB, Banks RE, Eusterman V, Brooks D. Analysis of vocal fold function in the miniswine model. J Invest Surg 1995;8:409-424.

6. Thibeault SL, Gray SD, Bless DM, Chan RW, Ford CN. Histologic and rheologic characterization of vocal fold scarring. J Voice 2002;16:96-104.

7. Rousseau B, Hirano S, Scheidt TD, Welham NV,Thibeault SL, Chan RW, Bless DM. Characterization of Vocal Fold Scarring in a Canine Model. Laryngoscope 2003;113:620627.

8. Hirano S, Bless DM, Rousseau B, Welham N, Scheidt T, Ford CN. Fibronectin and Adhesion Molecules on Canine Scarred Vocal Folds. Laryngoscope. 2003;113:966-972.

9. Thibeault SL, Rousseau B, Welham NV, Hirano S, Bless DM. Hyaluronan levels in acute vocal fold scar. Laryngoscope 2004;114:760-4.

10. Thibeault SL, Bless DM, Gray SD. Interstitial protein alterations in rabbit vocal fold with scar. J Voice. 2003;17:377-383.

11. Rousseau B, Sohn J, Montequin DW, Tateya I, Bless DM. Functional outcomes of reduced hyaluronan in acute vocal fold scar. Ann Otol Rhinol Laryngol 2004;113:767-76.

12. Tateya T, Tateya I, Sohn J, Bless DM. Histologic characterization of rat vocal fold scarring. 
Human scarred vocal folds 15

Ann Otol Rhinol Laryngol 2005;114:183-191.

13. Hirano M. Phonosurgery. Basic and clinical investigations. Otologia (Fukuoka) 1975;21:239440.

14. Remacle M, Eckel HE, Antonelli AR, et al. Endoscopic cordectomy. A proposal for a classification by the Working Committee, European Laryngological Society. Eur Arch Otorhinolaryngol 2000;257:227-31.

15. Gray SD, Titze IR, Chan R, Hammond TH. Vocal fold proteoglycans and their influence on biomechanics. Laryngoscope 1999;109:845-854.

16. Lehman JJ, Bless DM, Brandenburg JH. An objective assessment of voice production after radiation therapy for stage I squamous cell carcinoma of the glottis. Otolaryngol Head Neck Surg 1988;98:121-129.

17. Hahn MS, Kobler JB, Zeitels SM, Langer R. Midmembranous vocal fold lamina propria proteoglycans across selected species. Ann Otol Rhinol Laryngol 2005;114:451-62.

18. Savani R, Bagli DJ, Harrison RE, Turley EA. The role of hyaluronan-receptor interactions in wound repair. In: Garg HG, Longaker MT, eds. Scarless wound healing. New York, NY: Marcel Dekker; 2000; 115-137.

19. Chan RW, Titze IR. Hyaluronic acid (with fibronectin) as a bioimplant for the vocal fold mucosa. Laryngoscope 1999;109:1142-1149.

20. Grinnell F. Fibronectin and wound healing. J Cell Biochem 1984;26:107-116.

21. Bridger MW, Jahn AF, van Nostrand AW. Laryngeal rheumatoid arthritis. Laryngoscope 1980;90:296-303.

22. Speyer R, Speyer I, Heijnen MA. Prevalence and Relative Risk of Dysphonia in Rheumatoid Arthritis. J Voice. 2007 (in press).

23. Ehrlich HP. Collagen considerations in scarring and regenerative repair. In: Garg HG, Longaker MT eds. Scarless wound healing. Marcel Dekker Inc., New York, NY, 2000: 99113. 
24. Thibeault SL, Gray SD, Li W, Ford CN, Smith ME, Davis RK. Genotypic and phenotypic expression of vocal fold polyps and reinke's edema: A preliminary study. Ann Otol Rhinol Laryngol 2002;111:302-309. 
Human scarred vocal folds 17

\section{FIGURE LEGENDS}

Fig 1. Type I cordectomy scar (Case 1). (A) Elastica-von-Gieson stain (x 10). Collagen (stained red) is distributed almost throughout the lamina propria, being well organized. Elastin (stained black) exists in the deep portion, being well organized. (B, C) Alcian blue stain with or without HA digestion (x 10). Hyaluronic acid barely exists. (D) Immunohistochemistry for fibronectin (x 10). Fibronectin exists in the superficial portion. (E) Immunohistochemistry for decorin (x 10). Decorin deposits in the superficial portion.

Fig 2. Type I cordectomy scar (Case 2). (A) Elastica-von-Gieson stain (x 10). Mild deposition of collagen is noted by an arrow in the superficial portion. Elastin disappears. (B, C) Alcian blue stain with or without HA digestion (x 10). Hyaluronic acid exists widely in the lamina propria (B), which was totally digested by hyaluronidase (C). (D) Immunohistochemistry for fibronectin (x 10). Fibronectin is barely detected. (E) Immunohistochemistry for decorin (x 10). Decorin is detected as stained brown in the lamina propria.

Fig 3. Scar after type I cordectomy + radiotherapy (Case 4). (A) Elastica-von-Gieson stain (x 10). Excessive collagen deposition (stained red), forming thick bundles, with no elastin is noted throughout the lamina propria. (B, C) Alcian blue stain with or without HA digestion (x 10). Hyaluronic acid barely exists. (D) Immunohistochemistry for fibronectin (x 10). Fibronectin is barely stained. (E) Immunohistochemistry for decorin (x 10). Decorin is noted as brown stain.

Fig 4. Type II cordectomy scar (Case 8). (A) Elastica-von-Gieson stain (x 10). Excessive collagen deposition with no elastin is noted throughout the lamina propria. (B, C) Alcian blue stain with or without HA digestion (x 10). Hyaluronic acid barely exists. (D) Immunohistochemistry for fibronectin (x 10). Fibronectin is rare. (E) Immunohistochemistry for decorin (x 10). Decorin is not detected. 
Fig 5. Type III cordectomy scar (Case 9). (A) Elastica-von-Gieson stain (x 10). Excessive collagen deposition (stained red), forming thick bundles, is noted throughout the lamina propria. Elastin (black stain) is disorganized. (B, C) Alcian blue stain with or without HA digestion (x 10). Hyaluronic acid barely exists. (D) Immunohistochemistry for fibronectin (x 10). Fibronectin is barely stained. (E) Immunohistochemistry for decorin (x 10). Decorin is not noted.

Fig 6. Scar by rheumatoid arthritis + superficial cordotomy (Case 10). (A) Elastica-von-Gieson stain (x 10). Excessive collagen deposition (stained red), forming thick bundles, is noted throughout the lamina propria. Elastin disappears. $(\mathrm{B}, \mathrm{C})$ Alcian blue stain with or without HA digestion (x 10). Hyaluronic acid barely exists. (D) Immunohistochemistry for fibronectin (x 10). (E) Immunohistochemistry for decorin (x 10). Fibronectin and decorin is noted as brown stain. 


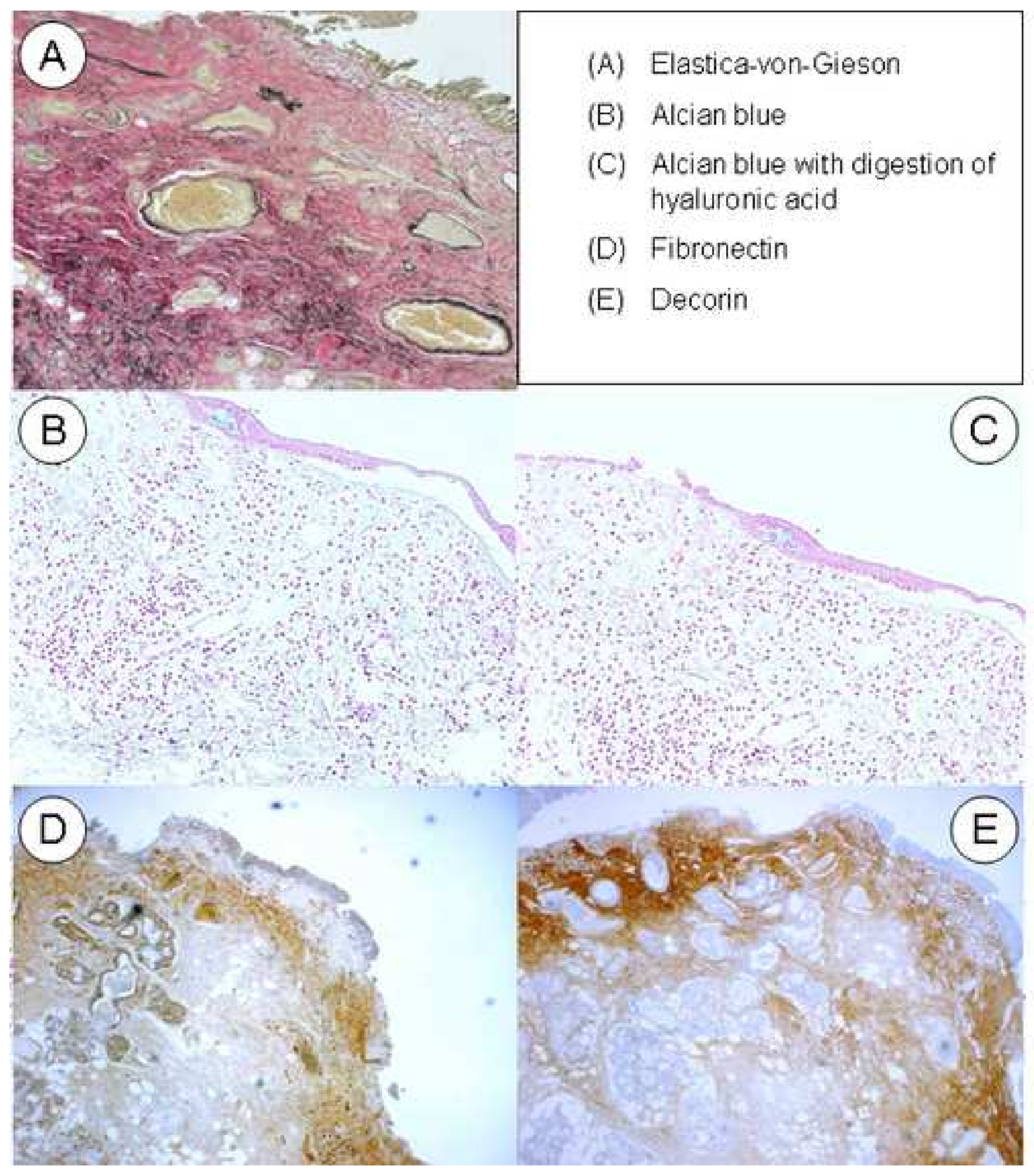




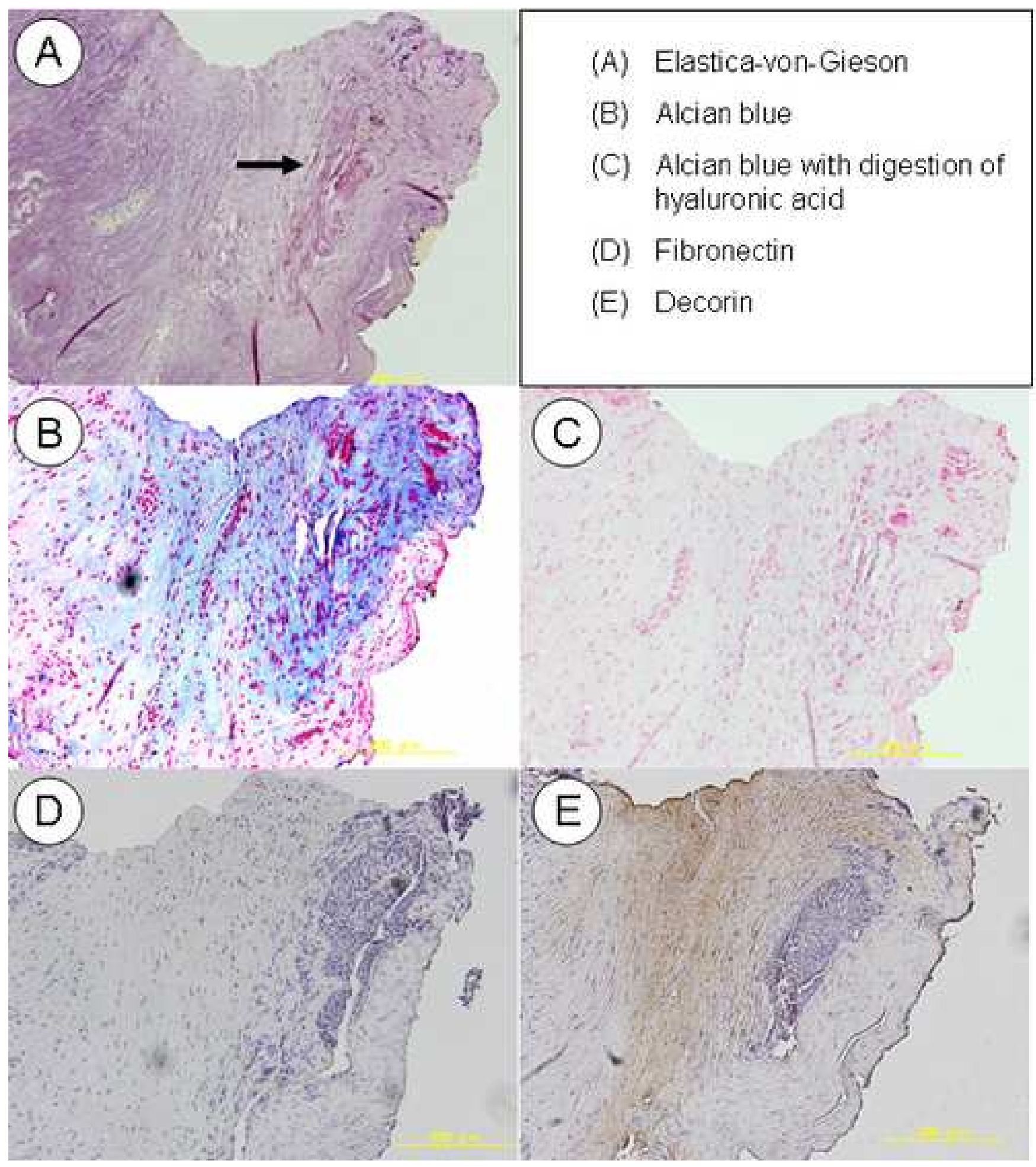




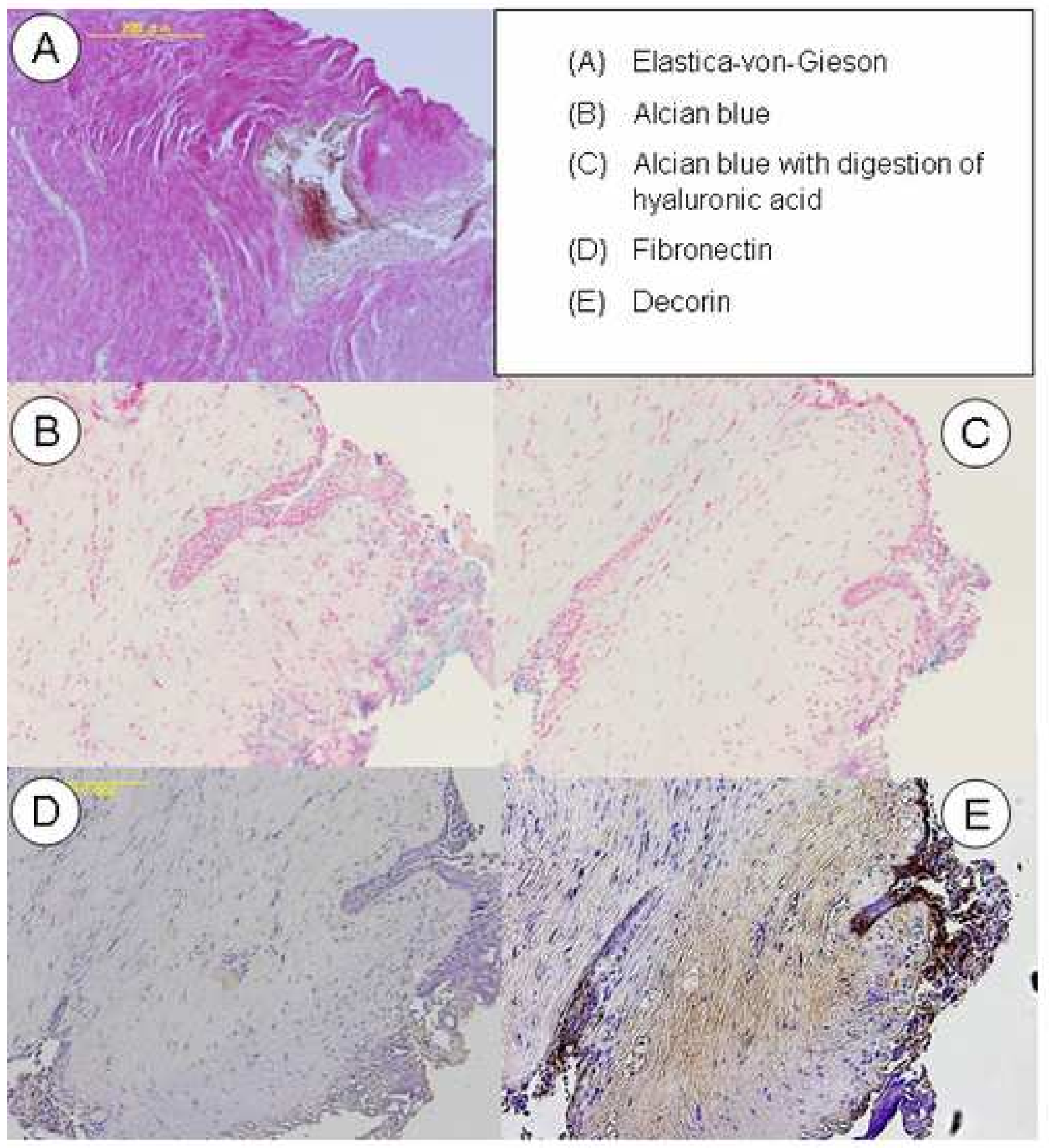




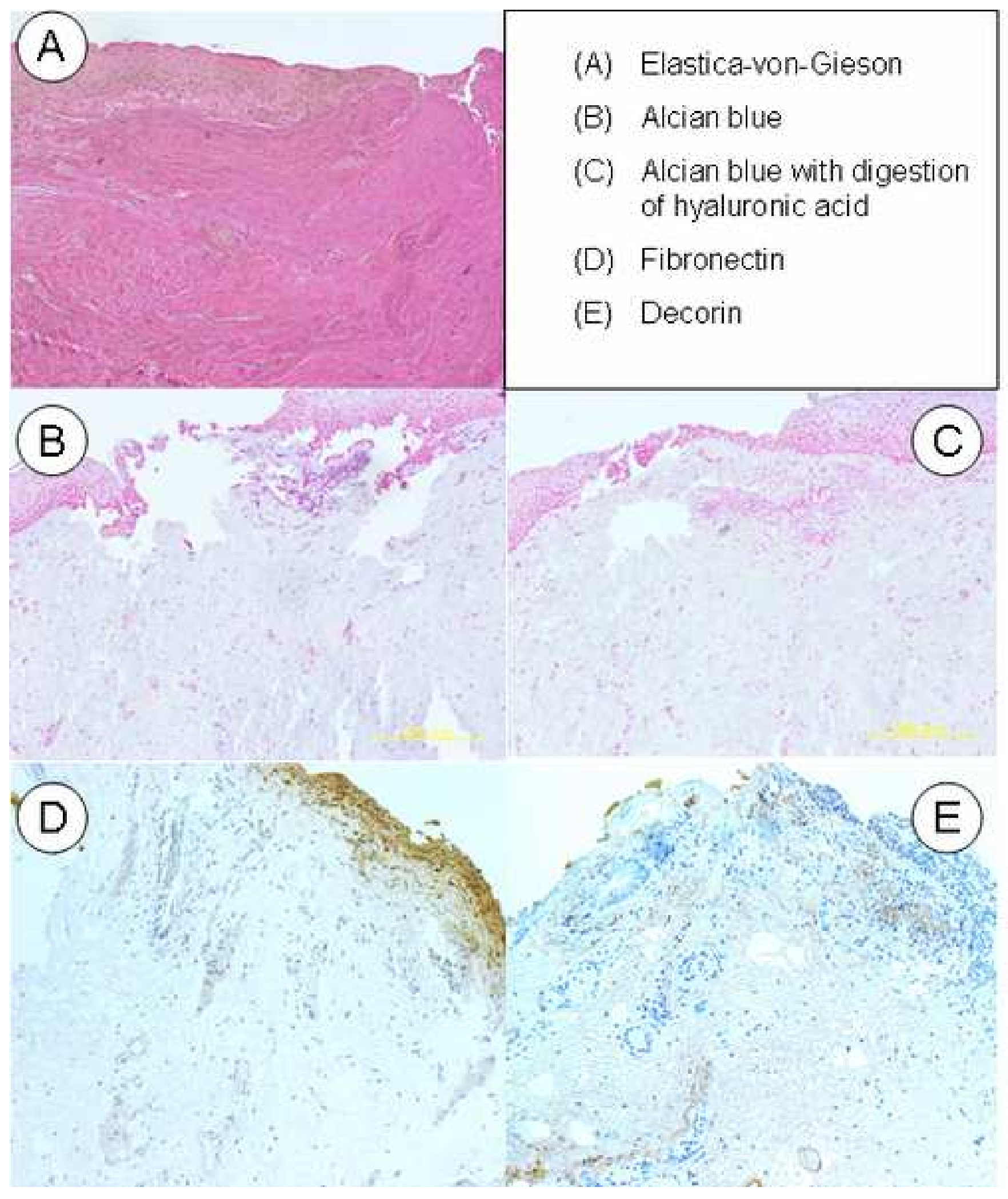




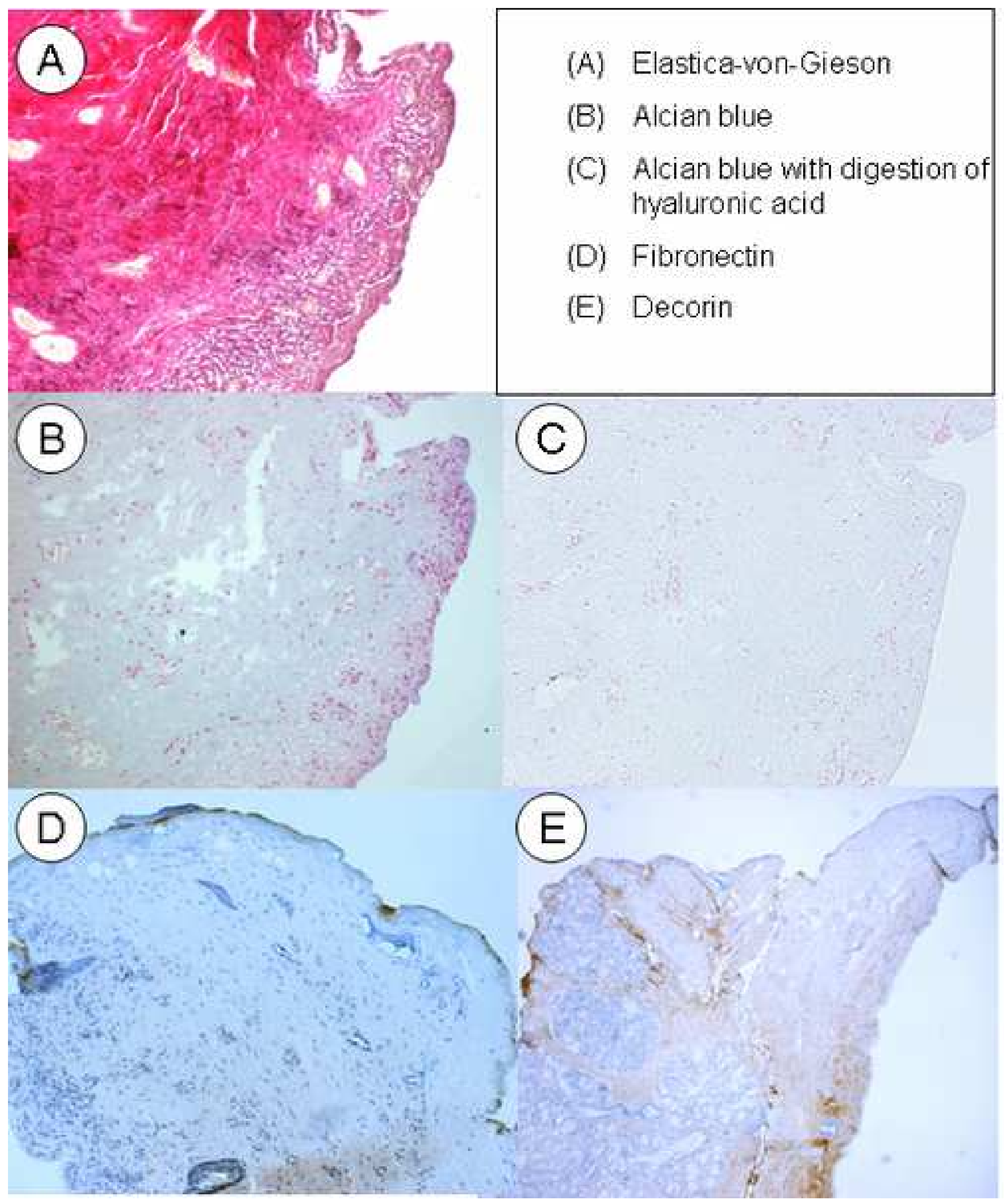




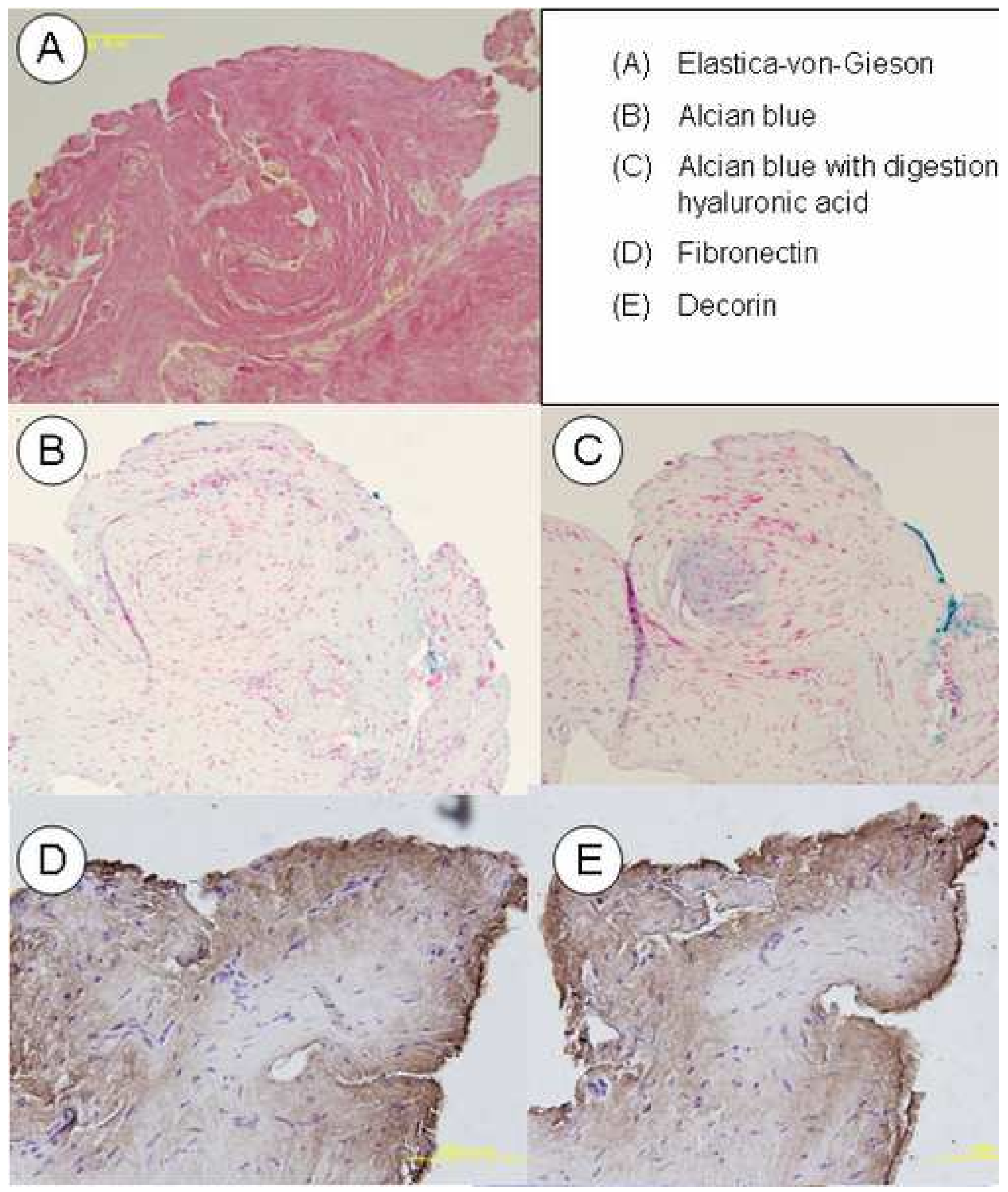


TABLE I. PATIENT CHARACTERISTICS

\begin{tabular}{cccccc}
\hline Case & Age & Gender & Etiology & $\begin{array}{c}\text { Cordectomy } \\
\text { type* }\end{array}$ & $\begin{array}{c}\text { Duration from cordectomy } \\
\text { to biopsy (months) }\end{array}$ \\
\hline 1 & 66 & Male & T1a & I & $3.5 \mathrm{mo}$ \\
2 & 55 & Male & T1a & I & $4 \mathrm{mo}$ \\
3 & 69 & Male & T1b & I & $3 \mathrm{mo}$ \\
4 & 54 & Male & T1a & I +RT & $7 \mathrm{mo}$ \\
5 & 68 & Male & T1a & II & $4 \mathrm{mo}$ \\
6 & 59 & Male & rT1a & II & $12 \mathrm{mo}$ \\
7 & 70 & Male & T1a & II & $4 \mathrm{mo}$ \\
8 & 58 & Male & T1a & II & $3 \mathrm{mo}$ \\
9 & 76 & Male & T1a & III & $13 \mathrm{mo}$ \\
10 & 40 & Female & RA & cordotomy & $3 \mathrm{mo}$ \\
\hline
\end{tabular}

*Type I: subepithelial, II: subligamental, III: transmuscular cordectomy

RT: radiotherapy, RA:reumatoid arthritis 
TABLE II. DEPOSITION OF EXTRACELLULAR MATRIX

\begin{tabular}{cccccccc}
\hline Case & $\begin{array}{c}\text { Cordectomy } \\
\text { type }\end{array}$ & $\begin{array}{c}\text { Time of } \\
\text { biopsy (mo) }\end{array}$ & Collagen & Elastin & $\begin{array}{c}\text { Hyaluronic } \\
\text { acid }\end{array}$ & Fibronectin & Decorin \\
\hline 1 & I & $3.5 \mathrm{mo}$ & mild & organized & - & + & + \\
2 & I & $4 \mathrm{mo}$ & mild & - & + & - & + \\
3 & I & $3 \mathrm{mo}$ & excessive & - & + & - & + \\
4 & I +RT & $7 \mathrm{mo}$ & excessive & - & - & - & + \\
5 & II & $4 \mathrm{mo}$ & excessive & - & + & - & - \\
6 & II & $12 \mathrm{mo}$ & excessive & disorganized & - & - & + \\
7 & II & $4 \mathrm{mo}$ & excessive & - & + & + & + \\
8 & II & $3 \mathrm{mo}$ & excessive & - & - & - & - \\
9 & III & $13 \mathrm{mo}$ & excessive & disorganized & - & - & - \\
10 & cordotomy & $3 \mathrm{mo}$ & excessive & - & - & + & + \\
\hline
\end{tabular}

